\title{
Controlling Oscillatory Behaviour of a Two Neuron Recurrent Neural Network Using Inputs
}

\author{
Robert Haschke, Jochen J. Steil, Helge Ritter \\ University of Bielefeld, Department of Computer Science, Neuroinformatics Group, \\ P.O.-Box 1001 31, D-33501 Bielefeld, Germany
}

\begin{abstract}
We derive analytical expressions of codim-1-bifurcations for a fully connected, additive two-neuron network with sigmoidal activations, where the two external inputs are regarded as bifurcation parameters. The obtained NeimarkSacker bifurcation curve encloses a region in input space with stable oscillatory behaviour, in which it is possible to control the oscillation frequency by adjusting the inputs.
\end{abstract}

\section{Introduction}

Since physiological experiments revealed that the brain exhibits complex oscillatory or even chaotic dynamics, research interest concerning the dynamical properties of artificial neural networks has increased. The experiments suggested that the brain intensively uses complex dynamics to solve its computational problems. And because convergent feedforward networks are relatively well understood, it is natural to investigate oscillatory dynamics of recurrent networks as the next level of complexity.

Because generally oscillatory dynamics of recurrent networks of reasonable size is difficult to describe analytically, several papers numerically proved the existence of a wide range of dynamical behaviour - from a fixed point over a quasiperiodic to a chaotic regime [1]. However, tools from bifurcation theory allow a mathematical analysis of fixed point bifurcations. Since two-neuron networks already exhibit quasiperiodic and chaotic dynamics [6] we restrict our analysis to such simple networks. Because of their low dimension these networks are tractable by analytical methods and thus can be used as well understood, basic building blocks of more complex networks.

Dynamical regimes of continuous-time and discrete-time networks with two varying parameters have been studied for various network architectures and nonlinearities. However, these works consider special connectivities [2] or are restricted to a numerical description [5]. In the present work we consider the external inputs as bifurcation parameters and derive analytical expressions for the bifurcation curves of saddle node, period doubling and Neimark-Sacker bifurcations in dependence of the weights of a fully connected additive network. Our results confirm and extend a numerical investigation of a two-neuron network made for the special case of no selfcoupling in [5]. Izhikevich performed a similar analysis for a continuous-time network, whose Hopf bifurcation curve differs from the Neimark-Sacker bifurcation curve [3].

The Neimark-Sacker curve encloses a region in input space where the network exhibits oscillatory behaviour. Thus crossing this curve allows to switch on and off oscillations. Furthermore input variations within this region allow frequency control. 
After introducing the investigated network model in section 2 we derive the bifurcation curves in section 3. The obtained expressions are discussed in section 4 with respect to different weight parameters and their corresponding dynamical regimes. Finally in section 5 we investigate the range, in which the oscillation frequency can vary in oscillatory regime. Section 6 concludes with a short discussion.

\section{Network Model}

We investigate the following class of discrete-time additive recurrent neural networks consisting of two neurons:

$$
\begin{aligned}
& s_{1} \mapsto F_{1}(s):=\sigma\left(w_{11} s_{1}+w_{12} s_{2}+u_{1}\right) \\
& s_{2} \mapsto F_{2}(s):=\sigma\left(w_{21} s_{1}+w_{22} s_{2}+u_{2}\right)
\end{aligned}
$$

where $s_{1}, s_{2}$ are the state variables of the system describing the activities of each neuron. The parameters $w_{i j}$ and $u_{i}$ represent the synaptic weight connecting neuron $j$ to neuron $i$ and the external input to neuron $i$ respectively. The activation function $\sigma$ is assumed to be sigmoid. Especially we consider tanh and Fermi function:

$$
\sigma_{\tanh }(x)=\tanh (x) \quad \sigma_{\text {fermi }}(x)=\left(1+\mathrm{e}^{-x}\right)^{-1}=\frac{1}{2}(\tanh (x / 2)+1)
$$

Note that the tanh and the Fermi system are topologically equivalent. Using the transformation $\hat{\boldsymbol{s}}=\frac{1}{2}(\boldsymbol{s}+\mathbf{1})$, where $\mathbf{1}=[1,1]^{t}$, one obtains for a tanh system: $\hat{\boldsymbol{s}} \mapsto$ $\boldsymbol{\sigma}_{\text {fermi }}(4 W \hat{\boldsymbol{s}}-2 W \mathbf{1}+2 \boldsymbol{u})$. With the inverse transformation the Fermi system can be written as: $\hat{\boldsymbol{s}} \mapsto \tanh \left(\frac{1}{4} W \hat{\boldsymbol{s}}+\frac{1}{2} W \mathbf{1}+\frac{1}{2} \boldsymbol{u}\right)$.

\section{Bifurcation Curves}

In the following we assume the weight parameters $w_{i j}$ to be fixed and the external, time-constant inputs $u_{i}$ as the bifurcation parameters. An input-dependent fixed point $\bar{s}:=\bar{s}(\boldsymbol{u})$ of the dynamics (1), i.e. $\boldsymbol{F}(\overline{\boldsymbol{s}})=\overline{\boldsymbol{s}}$, is asymptotically stable as long as all eigenvalues $\lambda$ of the Jacobian

$$
J:=J(\bar{s})=\left(D_{s} F\right)(\bar{s})=D(\bar{s}) W
$$

satisfy $|\lambda|<1 . D_{i j}:=\delta_{i j} \sigma^{\prime}\left(\bar{h}_{i}\right)$ is the diagonal matrix of derivatives $\sigma^{\prime}\left(\bar{h}_{i}\right)$ where $\overline{\boldsymbol{h}}=W \overline{\boldsymbol{s}}+\boldsymbol{u}$. The fixed point becomes unstable and the system undergoes a qualitative change of dynamics, if an eigenvalue $\lambda$ leaves the unit circle in the complex plane. Depending on this eigenvalue the following basic bifurcations are discriminated [4]: ${ }^{1}$

\begin{tabular}{llll} 
eigenvalue & bifurcation type & necessary condition & change of dynamics \\
\hline$\lambda=1$ & saddle node & $\operatorname{det}(J-\mathbf{1})=0$ & birth of two new fixed points \\
$\lambda=-1$ & period doubling & $\operatorname{det}(J+\mathbf{1})=0$ & birth of a 2-cycle \\
$\lambda=\mathrm{e}^{ \pm \mathrm{i} \theta}$ & Neimark-Sacker & $\operatorname{det}(J)=1$ & birth of a limit cycle
\end{tabular}

\footnotetext{
${ }^{1}$ As already mentioned continuous-time networks have the same bifurcation conditions only in case of saddle node bifurcations. Period doubling bifurcations do not occur and the necessary condition $\operatorname{tr} J=0$ for a Hopf bifurcation differs from its discrete-time pendant.
} 
In order to separate the input space into regions of qualitative different dynamics we have to calculate the bifurcation curves, which are separating manifolds of these regions. Thus along a bifurcation curve in the $\left(u_{1}, u_{2}\right)$-plane the fixed point equations

$$
\bar{s}_{i}=\sigma\left(\sum w_{i j} \bar{s}_{j}+u_{i}\right) \quad \Leftrightarrow \quad u_{i}=\sigma^{-1}\left(\bar{s}_{i}\right)-\sum w_{i j} \bar{s}_{j} \quad i=1,2
$$

together with the appropriate bifurcation condition hold. To find the bifurcation curves the corresponding system of three nonlinear equations - the bifurcation condition and the two fixed point equations (4) - can be solved due to a special property of the activation functions. More precisely we use the identities

$$
\begin{aligned}
& \sigma_{\text {tanh }}^{\prime}\left(\overline{h_{i}}\right)=1-\sigma_{\text {tanh }}^{2}\left(\overline{h_{i}}\right)=1-{\overline{s_{i}}}^{2} \\
& \sigma_{\text {fermi }}^{\prime}\left(\overline{h_{i}}\right)=\sigma_{\text {fermi }}\left(\overline{h_{i}}\right)\left(1-\sigma_{\text {fermi }}\left(\overline{h_{i}}\right)\right)=\overline{s_{i}}\left(1-\overline{s_{i}}\right)
\end{aligned}
$$

to solve each of the bifurcation conditions for $\bar{s}_{2}$ in dependence of $\bar{s}_{1}$. This is possible because the equations $(5,6)$ express the derivatives $\sigma^{\prime}$ in terms of the activation function itself and thus ensure together with the fixed point equations (4) that the bifurcation condition does not depend explicitly on the input parameters. Thus we get for the different bifurcation types:

tanh function

saddle node

$$
\bar{s}_{2}= \pm \sqrt{1+\frac{1-w_{11} x}{\operatorname{det} W x-w_{22}}}
$$

period doubling

$$
\bar{s}_{2}= \pm \sqrt{1+\frac{1+w_{11} x}{\operatorname{det} W x+w_{22}}}
$$

Neimark-Sacker $\quad \bar{s}_{2}= \pm \sqrt{1-\frac{1}{\operatorname{det} W x}}$
Fermi function

$$
\begin{aligned}
& \bar{s}_{2}=\frac{1}{2} \pm \sqrt{\frac{1}{4}+\frac{1-w_{11} x}{\operatorname{det} W x-w_{22}}} \\
& \bar{s}_{2}=\frac{1}{2} \pm \sqrt{\frac{1}{4}+\frac{1+w_{11} x}{\operatorname{det} W x+w_{22}}} \\
& \bar{s}_{2}=\frac{1}{2} \pm \sqrt{\frac{1}{4}-\frac{1}{\operatorname{det} W x}}
\end{aligned}
$$

where we used the abbreviations $x=1-\bar{s}_{1}^{2}$ in case of tanh function and $x=\bar{s}_{1}\left(1-\bar{s}_{1}\right)$ in case of Fermi function. Inserting this into the solved fixed point equations (4) we get the bifurcation curves in the $\left(u_{1}, u_{2}\right)$-plane parameterized with respect to $\bar{s}_{1}$ (see Fig. 1 ).

\section{Dynamical Regimes in Dependence of Weights}

First we discuss which parameter ranges can exhibit interesting dynamical behaviour. As long as $\sup _{\bar{s}}\|J(\bar{s})\| \leq \sup _{\bar{s}}\|D(\bar{s})\|\|W\|<1$ the system's mapping $F$ is a contraction, so that a unique globally asymptotic stable fixed point exists. This condition especially holds for a small norm $\|W\| \ll 1$ and for large amplitudes of the inputs $u_{i}$. In the latter case the activations $\overline{\boldsymbol{h}}=W \overline{\boldsymbol{s}}+\boldsymbol{u}$ are in the saturation region of the sigmoid, such that the diagonal matrix $D$ of derivatives $\sigma^{\prime}\left(\bar{h}_{i}\right)$ becomes small in magnitude. Thus interesting dynamical behaviour with more than one stable fixed point can be expected for relatively small inputs and weight matrices which satisfy $\|W\| \geq 1$.

Oscillations of arbitrary frequency $\omega$ arise, if a Neimark-Sacker bifurcation occurs, whereby the angular frequency $\omega \approx \theta$ is in first order given by the argument of the 


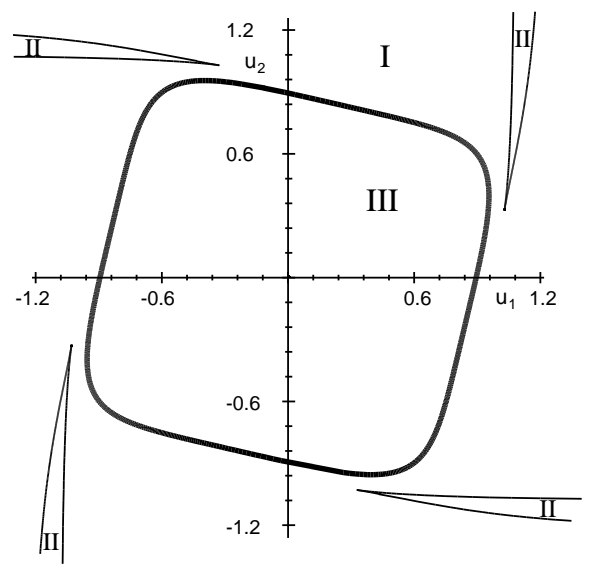

(a) $\tanh , w_{i i}=1.23, w_{21}=-w_{12}=1.15$

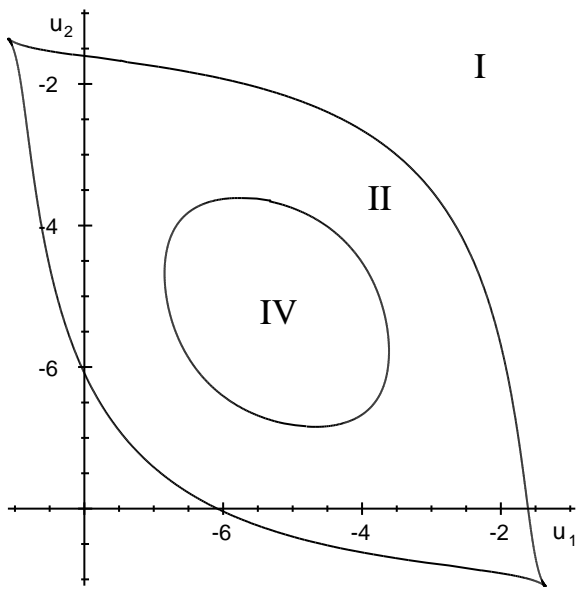

(b) Fermi, $w_{i i}=3, w_{12}=w_{21}=7.45$

Fig. 1. Computed bifurcation curves for tanh function (a) and Fermi function (b) for different weight parameters $w_{i j}$. Region I corresponds to a globally asymptotic stable fixed point. In region II there exist three fixed points, two of them stable and one unstable. Region III corresponds to oscillatory behaviour evoked by a Neimark-Sacker bifurcation (bold curve) contrasting to region $\mathrm{IV}$, which corresponds to a 2-cycle, evoked by a period doubling bifurcation. Note that due to the topological equivalence of the tanh and Fermi system all bifurcations curves can be observed in both systems, of course with appropriate changes of weights and inputs.

complex Jacobian eigenvalues $\lambda(J)=\mathrm{e}^{ \pm \mathrm{i} \theta}$. If we consider the tanh function with zero inputs, the system's Jacobian at the origin reduces to $J(0)=W$. This allows to choose an appropriate weight matrix with respect to a desired oscillation frequency $\omega$ for $\boldsymbol{u}=0$. This result still holds for inputs belonging to a small neighbourhood of the origin, because eigenvalues continuously depend on matrix entries.

Figure 1 shows the computed bifurcation curves for tanh and Fermi systems at different weight parameters, which are chosen according to the previous considerations. In all subfigures region I corresponds to a globally asymptotic regime of a unique fixed point. As expected, this region always includes parameter ranges, where both inputs $u_{1}$ and $u_{2}$ are large in magnitude. Because there exist no other bifurcation curves in this region and our expressions cover all possible fixed point bifurcations, the uniqueness of the fixed point is guaranteed. Its existence follows from Brouwer's Fixed Point Theorem.

Region II marks a regime of three fixed points, two of them stable and one unstable. If the input parameters are varied quasi-statically - i.e. slowly enough that the system always approaches a limit set - along a curve crossing region II, a hysteresis effect occurs, as is discussed in [5]. The singular point, where both saddle node bifurcations curves meet, corresponds to a cusp bifurcation, at which all three fixed points coalesce.

In region III an unstable fixed point and a stable limit cycle exist. This cycle is evoked by a Neimark-Sacker bifurcation contrasting to region IV, where a stable 2- 
cycle - evoked by a period doubling bifurcation - exists beside the unstable fixed point. As is shown in [1] region III exhibits a wide range of complex dynamical behaviour. There exist sub-regions where frequency locking occurs, i.e. the quasiperiodic limit cycle reduces to a stable $k$-cycle. Furthermore special singular weight matrices produce chaotic dynamics in region III, as proven by Wang [6].

Note that the bifurcation curves of saddle node and period-doubling become identical if no self-coupling is allowed. Thus crossing the bifurcation curve besides the existing stable fixed point two new fixed points (a stable and an unstable one) occur together with a stable period-2 orbit. Because $x \geq 0$ and $\operatorname{det} W=w_{12} w_{21}$ in this case, saddle node and period-doubling bifurcations occur only for even, Neimark-Sacker bifurcations only for odd 2-modules. (The bifurcation curves exist only if the derived expressions for $\bar{s}_{2}$ are defined and assume values within $[0 . .1]$ or $[-1 . .1]$ for fermi resp. tanh function. This result confirms numerically obtained results of Pasemann [5].

\section{Frequency Control}

As discussed in previous section, crossing the Neimark-Sacker bifurcation curve into region III produces a stable limit cycle, whose frequency equals up to first order the argument of the Jacobian eigenvalue $\lambda(J(\bar{s}))$ on the bifurcation curve. Generally the eigenvalues change along the curve and so does the frequency. Furthermore the oscillation frequency changes, if the input parameters move deeper into region III, because of nonlinear effects.

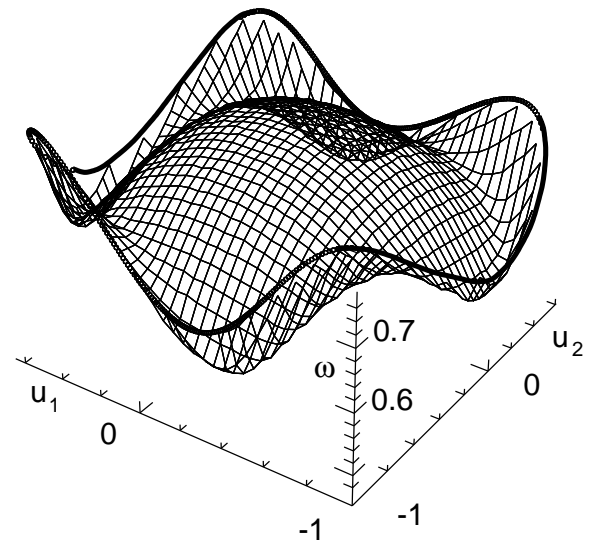

Fig. 2. Frequency drift within region III of neural network of figure 1a.

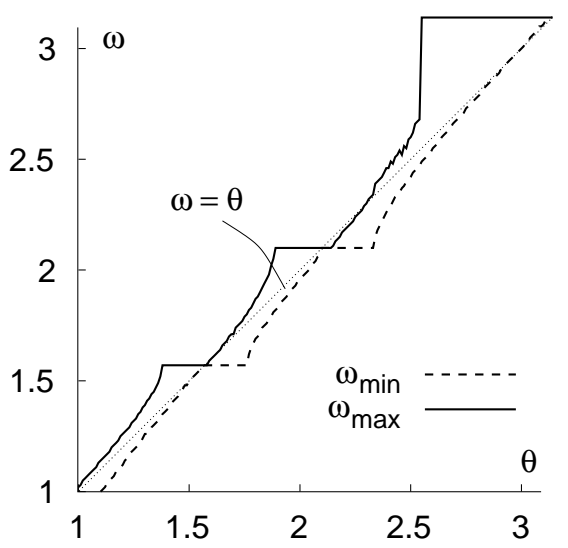

Fig. 3. Frequency range in dependence of rotation angle $\theta$ of weight matrix.

Figure 2 shows this frequency drift for a typical configuration with weight parameters equal to those of Figure 1a. The frequencies along the border of the grid are computed using the Jacobian eigenvalues. The frequencies inside the oscillatory region are 
obtained numerically as the strongest frequency component of a discrete Fourier transformation. As can be seen, they vary within a small range, such that it is possible to adjust the frequency choosing appropriate inputs. Choosing inputs outside the oscillatory region one can switch off oscillations completely.

To investigate the dependence of the frequency range on the weight parameters, we use matrices $W(g, \theta)$ possessing complex eigenvalues $\lambda=g \mathrm{e}^{ \pm \mathrm{i} \theta}$. Numerical studies reveal that the overall width of the possible frequency range increases with the gain $g$ and depends crucial on $\theta$, as shown in Figure 3. The minimally and maximally observable frequencies $\omega$ vary around the medium frequency $\theta$. The steps of these curves arise due to frequency locking to $\omega=\frac{1}{2} \pi, \frac{2}{3} \pi$ and $\pi$, which corresponds to the $1: 4,1: 3$ and $1: 2$ strong resonance respectively.

\section{Discussion}

We presented analytical expressions for bifurcation curves in the input space of discretetime two-neuron networks. These expressions enable us to switch on and off oscillatory behaviour by choosing appropriate inputs. Moving quasi-statically within the oscillatory region of the input space we can also continuously modify the frequency of the ongoing oscillation around a medium value defined by the weights. Such networks could be used as pattern generators or basic components of more complex networks, which would have higher-order information processing capabilities using resonance and synchronization effects between its components or with respect to time-dependent inputs. The far goal is to control the dynamics of large networks consisting of small modules using a small number of (input) parameters.

However, even in the simple case of a two-neuron network the whole range of dynamical behaviour cannot be described through the investigation of these basic bifurcations. Especially an investigation of the chaotic regime within region III of oscillatory behaviour requires the development of further mathematical tools.

Acknowledgments: This work was supported by the DFG grants GK-231.

\section{References}

1. E. Dauce, M. Quoy, B. Cessac, B. Doyon, and M. Samuelides. Self-organization and dynamics reduction in recurrent networks: stimulus presentation and learning. Neural Networks, 11:521-533, 1998.

2. M. di Marco, A. Tesi, and M. Forti. Bifurcations and oscillatory behaviour in a class of competitive cellular neural networks. International Journal of Bifurcation and Chaos, 10(6):12671293, 2000.

3. Frank C. Hoppensteadt and Eugene M. Izhikevich. Weakly Connected Neural Networks. Springer, New York, applied mathematical sciences edition, 1997.

4. Yuri A. Kuznetsov. Elements of Applied Bifurcation Theory, volume 112 of Applied Mathematical Sciences. Springer-Verlag, New York, Berlin, Heidelberg, 1995.

5. Frank Pasemann. Discrete dynamics of two neuron networks. Open Systems \& Information Dynamics, 2(1):49-66, 1993.

6. Xin Wang. Period-doublings to chaos in a simple neural network: An analytic proof. Complex Systems, 5:425 - 441, 1991. 